\title{
NLO QCD analysis of single-diffractive dijet production at the Tevatron
}

\author{
Michael Klasen* \\ Laboratoire de Physique Subatomique et de Cosmologie, Université Joseph Fourier I \\ CNRS-IN2P3 / INPG, 53 Avenue des Martyrs, F-38026 Grenoble, France \\ E-mail: klasen@lpsc.in2p3.fr
}

\begin{abstract}
We present the first NLO QCD analysis of single-diffractive dijet production in proton-antiproton collisions. By comparing the ratio of single- and non-diffractive cross sections to data from the Tevatron, the rapidity-gap survival probability is determined as a function of the momentum fraction of the parton in the antiproton. Assuming Regge factorization, this probability can be interpreted as a suppression factor for the diffractive structure function measured in deep-inelastic scattering at HERA. In contrast to the observations for photoproduction, the suppression factor in proton-antiproton collisions depends on the momentum fraction of the parton in the Pomeron even at NLO.
\end{abstract}

XVIII International Workshop on Deep-Inelastic Scattering and Related Subjects April 19 -23, 2010

Convitto della Calza, Firenze, Italy

${ }^{*}$ Speaker. 


\section{Introduction}

In high-energy hadronic collisions, a large fraction of the events is produced diffractively and contains one or more leading (anti-)protons or excited hadronic states $Y$ with relatively low mass $M_{Y}$, which are separated from the central hard process by rapidity gaps. Hard diffraction has been studied intensely in deep-inelastic ep scattering (DIS) at HERA, where it can be understood due to the presence of the large squared photon virtuality $Q^{2}$ in terms of the Pomeron structure function $F_{2}^{D}\left(x, Q^{2}, \xi, t\right)=\sum_{i} f_{i / p}^{D} \otimes \sigma_{\gamma^{*} i}$, factorizing into diffractive parton density functions (DPDFs) $f_{i / p}^{D}$ and perturbatively calculable partonic cross sections $\sigma_{\gamma^{*} i}[1]$. Assuming in addition Regge factorization, the DPDFs $f_{i / p}^{D}=f_{i / \mathbb{P}}\left(\beta=x / \xi, Q^{2}\right) f_{\mathbb{P} / p}(\xi, t)$ can be parameterized in terms of a Pomeron flux factor $f_{\mathbb{P} / p}$, which depends on the longitudinal momentum fraction of the pomeron in the proton $\xi$ and the squared four-momentum transfer at the proton vertex $t$, and PDFs in the Pomeron $f_{i / \mathbb{P}}$, which depend on the momentum fraction of the partons in the pomeron $\beta$ and an unphysical factorization scale $Q^{2}$, and fitted to diffractive DIS data [2]. Diffractive dijet production data add further constraints, in particular on the gluon distribution [3]. At high $\xi$, a subleading Reggeon contribution must be taken into account.

As $Q^{2}$ decreases, the applicability of perturbative QCD must be ensured by sufficiently large jet transverse energies $E_{T 1,2}$. From the absorption of collinear initial-state singularities at next-toleading order (NLO) [4], the photon develops a hadronic structure and contributes not only with direct, but also resolved processes [5]. These processes occur also in hadron-hadron scattering, where factorization is broken due to the presence of soft interactions in both the initial and final states [6], and factorization breaking is indeed observed in dijet photoproduction at NLO [7]. Similar effects may also arise in dijet production with a leading neutron [8]. Soft rescattering must be understood, if one wishes to exploit processes like diffractive Higgs production, which offers a promising alternative to the difficult inclusive diphoton signal in the low-mass region [9]. In this article, we present the first NLO analysis of diffractive dijet production in hadron collisions [10].

\section{Diffractive dijet production at the Tevatron}

Dijet production with a leading antiproton has been measured by the CDF collaboration at the Tevatron in the range $|t|<1 \mathrm{GeV}^{2}$, used also by the $\mathrm{H} 1$ collaboration for the extraction of their PDFs 2006 Fit A,B and 2007 Fit Jets, but at slightly larger $\xi \in[0.035 ; 0.095]$ [11]. From the ratio $\tilde{R}\left(x_{\bar{p}}=\beta \xi\right.$ ) of single-diffractive (SD) to non-diffractive (ND) cross sections as a function of $x_{\bar{p}}=\sum_{i} E_{T i} / \sqrt{s} e^{-\eta_{i}}$, integrated over $E_{T i}>7 \mathrm{GeV}$ and $\left|\eta_{i}\right|<4.2$, an effective diffractive structure function $\tilde{F}_{J J}^{D}(\beta)=\tilde{R} \times F_{J J}^{N D}$ was extracted assuming $F_{J J}^{N D}\left(x, Q^{2}\right)=x\left[f_{g / p}+4 / 9 \sum_{i} f_{q_{i} / p}\right]$ with GRV 98 LO proton PDFs and compared to older H1 diffractive structure functions at fixed $Q^{2}=75$ $\mathrm{GeV}^{2}$. These were found to overestimate the measured diffractive structure function by about an order of magnitude, while the correction to be applied to the $\mathrm{H} 1$ measurements with $M_{Y}<1.6 \mathrm{GeV}$, when compared to the CDF measurements with leading antiprotons, should only be about 23\% [2].

In a subsequent publication, the CDF collaboration analyzed in a similar way data at $\sqrt{s}=$ $630 \mathrm{GeV}$, not only $1800 \mathrm{GeV}$, in order to test factorization, Pomeron flux renormalization, and rapidity-gap survival models [12], but found no significant energy dependence [13]. In addition they restricted the $|t|$-range to below $0.2 \mathrm{GeV}^{2}$ and the average transverse jet energy to $\bar{E}_{T}>10$ 

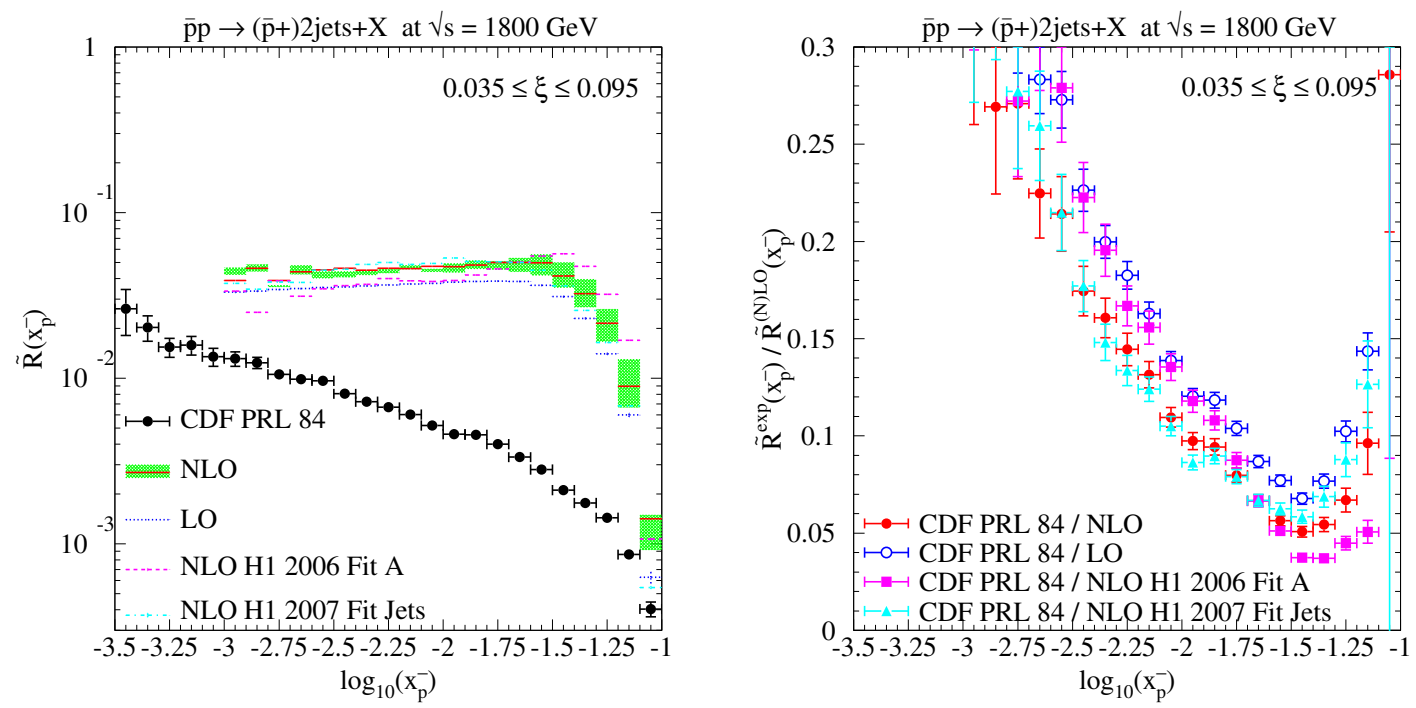

Figure 1: Left: Ratio $\tilde{R}$ of SD to ND dijet cross sections as a function of the momentum fraction of the parton in the antiproton, computed at NLO (with three different DPDFs) and at LO and compared to the Tevatron Run I data from the CDF collaboration. Right: Double ratio of experimental over theoretical values of $\tilde{R}$, equivalent to the factorization-breaking suppression factor required for an accurate theoretical description of the data (color online).

$\mathrm{GeV}$, which has the advantage of removing the infrared sensitivity of two equal cuts on $E_{T 1,2}$ [14]. In both publications, the extraction of $\tilde{F}_{J J}^{D}$ was performed on the basis of leading order (LO) QCD and assuming that the $t$-channel gluon exchange in the partonic cross sections dominates.

\section{NLO QCD analysis}

Our NLO QCD analysis is based on previous work on inclusive dijet photoproduction [15], where the resolved photon contribution can be directly applied to dijet hadroproduction [16]. It takes into account the convolution of Pomeron flux factors and PDFs, modern CTEQ6.6M proton and H1 2006 and 2007 Pomeron PDFs with $Q^{2}=E_{T 1}^{2}$ on an event-by-event basis, and the complete set of parton-parton scattering cross sections. Jets are defined by a cone with radius $R=0.7$ as in the CDF experiment and imposing a parton separation of $R_{\text {sep }}=1.3 R$. The normalized control distributions $1 / \sigma \mathrm{d} \sigma / \mathrm{d} \bar{E}_{T}$ and $1 / \sigma \mathrm{d} \sigma / \mathrm{d} \bar{\eta}$ (not shown) are in good agreement with the SD and ND measurements, in particular for $E_{T 2}>6.6 \mathrm{GeV}$ removing the infrared sensitivity in the first CDF analysis and for $\sqrt{s}=630 \mathrm{GeV}$ in the second CDF analysis [10]. However, the ratio $\tilde{R}$ of SD to ND dijet cross sections as a function of $x_{\bar{p}}$ shown in Fig. 1 (left) for $\sqrt{s}=1800 \mathrm{TeV}$ and $|t|<1 \mathrm{GeV}^{2}$ significantly overestimates the data for all employed diffractive PDFs and even more at NLO than at $\mathrm{LO}$, due to an average ratio of SD to ND $K$-factors of $1.35(1.6$ at $\sqrt{s}=630 \mathrm{GeV})$. Remember that all theoretical predictions should be divided by a factor of 1.23 to correct for diffractive dissociation included in the H1 DPDFs.

The double ratios $\tilde{R}^{\exp } / \tilde{R}^{(\mathrm{N}) \text { LO }}$ in Fig. 1 (right) and Fig. 2 can be interpreted as suppression factors or rapidity-gap survival probabilities, which are smaller than one due to soft rescattering. 

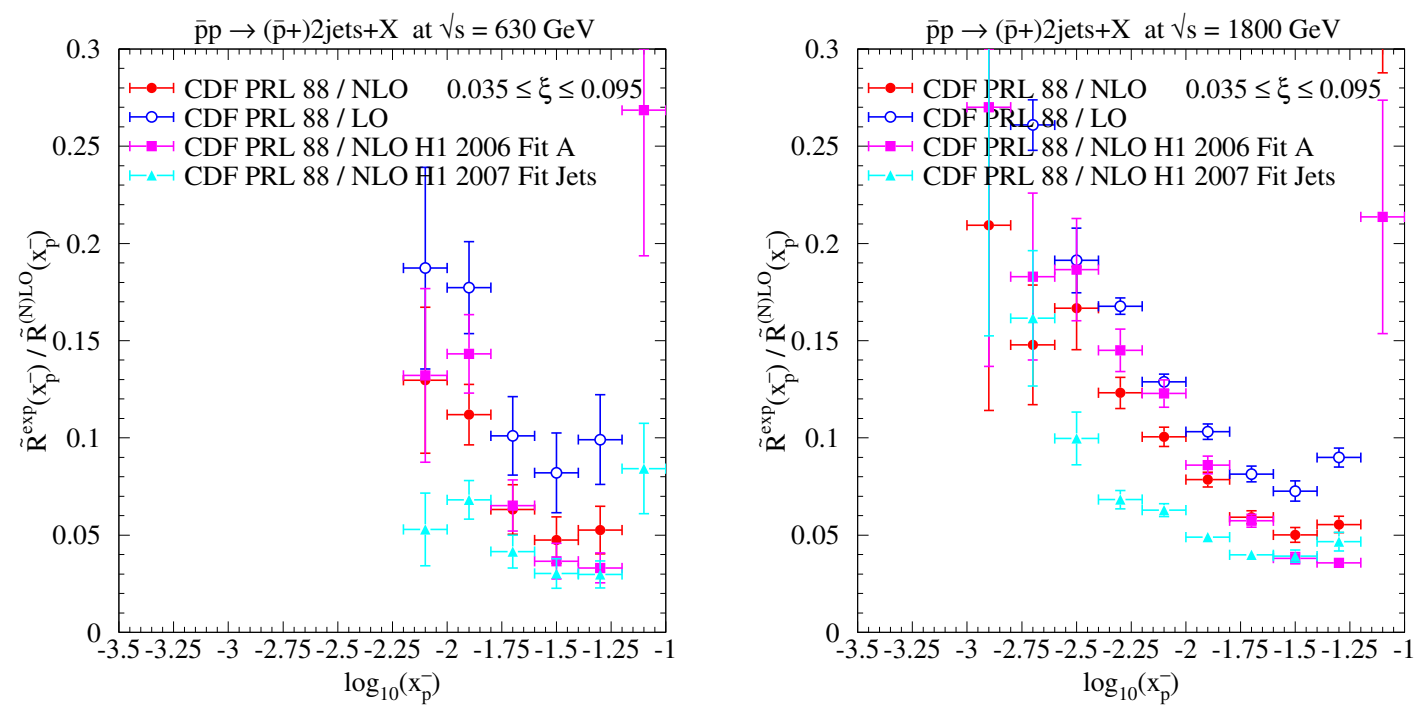

Figure 2: Double ratios of experimental over theoretical values of $\tilde{R}$, equivalent to the factorization-breaking suppression factor required for an accurate theoretical description of the data from the Tevatron at $\sqrt{s}=630$ (left) and $1800 \mathrm{GeV}$ (right) (color online).

Their qualitative behavior in these three figures is very similar: we observe a dependence on $x_{\bar{p}}$ with a minimum at $\log _{10}\left(x_{\bar{p}}\right) \simeq-1.5\left(x_{\bar{p}} \simeq 0.032\right)$, a rise towards smaller $x_{\bar{p}}$ by up to a factor of five, a smaller rise towards larger $x_{\bar{p}}$, and an appreciable dependence of the suppression factor on the chosen diffractive PDFs. The corresponding suppression factors for $\tilde{F}_{J J}^{D}(\beta)$ (not shown), computed with the same assumptions on $F_{J J}^{N D}$ as those taken by $\mathrm{CDF}$, are minimal with the value $\sim 0.05$ at $\beta=0.5$ and rise to $\sim 0.12$ for $\sqrt{s}=1800 \mathrm{GeV}$ and to $\sim 0.1$ for $\sqrt{s}=630 \mathrm{GeV}$ at $\beta=0.1$ and with H1 2006 Fit B. Here, the effect of NLO corrections is partially compensated by the simplifications inherent in the calculation of $\tilde{F}_{J J}^{D}$. The strong rise of the suppression factors to values of $\sim 0.25$ for $\beta<0.05\left[\log _{10}\left(x_{\bar{p}}\right)<-2.5\right]$ at $\sqrt{s}=1800 \mathrm{GeV}$ may not be conclusive, since the H1 DPDFs are largely unconstrained in this region. On the other hand, the rise at $\log _{10}\left(x_{\bar{p}}\right)>-1.5$ can be attributed to the Reggeon contribution, that should be added at large $\xi$. Note also that the variation of the suppression factor is considerably reduced for the H1 2007 Fit Jets, which has been obtained with additional constraints from diffractive DIS dijet production.

The extraction of $\tilde{F}_{J J}^{D}(\beta)$ from $\tilde{R}\left(x_{\bar{p}}\right)$ is based on the assumption that the latter is only weakly $\xi$-dependent and can be evaluated at an average value of $\bar{\xi}=0.0631$. This weak $\xi$-dependence is indeed observed in the newer CDF data and also in our theoretical calculations, which reflect the $\xi$-dependence of the $\mathrm{H} 1$ fits to the Pomeron flux factors $f_{\mathbb{P} / \bar{p}}(\xi, t) \propto \xi^{-m}$ with $m \simeq 1.1(0.9$ in the CDF fit to their data). The (small) difference of the theoretical (1.1) and experimental (0.9) values of $m$ can be explained by a subleading Reggeon contribution, which has not been included in our predictions. To study its importance, we have computed the ratio of the Reggeon over the Pomeron contribution to the LO single-diffractive cross section at $\sqrt{s}=1800 \mathrm{GeV}$. The Reggeon flux factor was obtained from H1 2006 Fit B and convolved, as it was done in this fit, with parton densities in the pion [17]. Very similar results were obtained for the H1 2007 Fit Jets Reggeon flux. On average, the Reggeon adds a 5\% contribution to the single-diffractive cross section, which is 
smaller at small $x_{\bar{p}}=\xi \beta$ and $\xi$ (2.5\%) than at large $x_{\bar{p}}$ and $\xi(8 \%)$. This corresponds to the graphs shown in Figs. 5 ( $\xi=0.01)$ and $6(\xi=0.03)$ of Ref. [2], e.g. at $Q^{2}=90 \mathrm{GeV}^{2}$. While the Reggeon contribution thus increases the diffractive cross section and reduces the suppression factor at large $x_{\bar{p}}$, making the latter more constant, the same is less true at small values of $x_{\bar{p}}$.

Any model calculation of the suppression factor or rapidity-gap survival probability must try to explain two points, first the amount of suppression, which is $\sim 0.1$ at $\beta=0.1$, and second its dependence on the variable $\beta$ ( or $x_{\bar{p}}$ ). Such a calculation has been performed by Kaidalov et al. [12]. In this calculation, the hard scattering cross section for the diffractive production of dijets was supplemented by screening or absorptive corrections on the basis of eikonal corrections in impact parameter space. The parameters of the eikonal were obtained from a two-channel description of high-energy inelastic diffraction. The exponentiation of the eikonal stands for the exchange of multi-Pomeron contributions, which violate Regge and QCD factorization and modify the predictions based on single Pomeron and/or Regge exchange. The obtained suppression factor is not universal, but depends on the details of the hard subprocess as well as on the kinematic configurations. The first important observation from this calculation is that in the Tevatron dijet analysis the mass squared of the produced dijet system $M_{J J}^{2}=x_{p} \beta \xi_{s}$ as well as $\xi$ are almost constant, so that small $\beta$ implies large $x_{p}$. The second important ingredient in this calculation is the assumption that the absorption cross section of the valence and the sea components, where the latter includes the gluon, of the incoming proton are different, in particular, that the valence and sea components correspond to smaller and larger absorption. For large $x_{p}$ or small $\beta$, the valence quark contribution dominates, which produces smaller absorptive cross sections as compared to the sea quark and gluon contributions, which dominate at small $x_{p}$. Hence the survival probability increases as $x_{p}$ increases and $\beta$ decreases. The convolution of the $\beta$-dependent absorption corrections with older $\mathrm{H} 1$ DPDFs [18] led to a prediction for $F_{J J}^{D}(\beta)$, which was in very good agreement with the corresponding experimental distribution (see Fig. 4 in [12]). A similar correction for soft rescattering of our single-diffractive NLO cross sections based on the more recent DPDFs of H1 should lead to a very similar result. An alternative model for the calculation of the suppression factor was developed by Gotsman et al. [19]. However, these authors did not convolve their suppression mechanism with the hard scattering cross section. Therefore a direct comparison to the CDF data is not possible.

At variance with the above discussion of diffractive dijet production in hadron-hadron scattering, the survival probability in diffractive dijet photoproduction was found to be larger $(\sim 0.5$ for global suppression, $\sim 0.3$ for resolved photon suppression only) and fairly independent of $\beta$ $[7,20]$. This can be explained by the fact that the HERA analyses are restricted to large values of $x_{\gamma} \geq 0.1$ (as opposed to small and intermediate values of $x_{p}=0.02 \ldots 0.2$ at the Tevatron), where direct photons or their fluctuations into perturbative or vector meson-like valence quarks dominate. The larger suppression factor in photoproduction corresponds also to the smaller center-of-mass energy available at HERA.

\section{Acknowledgments}

It is a pleasure to thank G. Kramer for his collaboration and comments on the manuscript, H. Abramowicz, A. Levy, and A. Valkarova for interesting discussions, and the organizers of the DIS 2010 conference for creating a stimulating scientific atmosphere despite adverse forces majeures. 


\section{References}

[1] J. C. Collins, Phys. Rev. D 57 (1998) 3051 [Erratum-ibid. D 61 (2000) 019902].

[2] A. Aktas et al. [H1 Collaboration], Eur. Phys. J. C 48 (2006) 715.

[3] A. Aktas et al. [H1 Collaboration], JHEP 0710 (2007) 042; S. Chekanov et al. [ZEUS Collaboration], Nucl. Phys. B 831 (2010) 1.

[4] M. Klasen and G. Kramer, J. Phys. G 31 (2005) 1391.

[5] M. Klasen and G. Kramer, Phys. Rev. Lett. 93 (2004) 232002.

[6] J. C. Collins, L. Frankfurt and M. Strikman, Phys. Lett. B 307 (1993) 161.

[7] M. Klasen and G. Kramer, Eur. Phys. J. C 38 (2004) 93; M. Klasen and G. Kramer, Mod. Phys. Lett. A 23 (2008) 1885 and references therein.

[8] M. Klasen and G. Kramer, Phys. Lett. B 508 (2001) 259; M. Klasen, J. Phys. G 28 (2002) 1091; M. Klasen and G. Kramer, Eur. Phys. J. C 49 (2007) 957.

[9] C. Royon, Mod. Phys. Lett. A 18 (2003) 2169.

[10] M. Klasen and G. Kramer, Phys. Rev. D 80 (2009) 074006.

[11] A. A. Affolder et al. [CDF Collaboration], Phys. Rev. Lett. 84 (2000) 5043.

[12] A. B. Kaidalov, V. A. Khoze, A. D. Martin and M. G. Ryskin, Eur. Phys. J. C 21 (2001) 521.

[13] A. A. Affolder et al. [CDF Collaboration], Phys. Rev. Lett. 88 (2002) 151802.

[14] M. Klasen and G. Kramer, Phys. Lett. B 366 (1996) 385.

[15] M. Klasen and G. Kramer, Z. Phys. C 76 (1997) 67; M. Klasen, T. Kleinwort and G. Kramer, Eur. Phys. J. direct C 1 (1998) 1.

[16] M. Klasen and G. Kramer, Phys. Lett. B 386 (1996) 384; M. Klasen and G. Kramer, Phys. Rev. D 56 (1997) 2702.

[17] J. F. Owens, Phys. Rev. D 30 (1984) 943.

[18] H1 Collaboration, T. Ahmed et al., Phys. Lett. B 348 (1995) 681; H1 Collaboration, C. Adloff et al., Z. Phys. C 76 (1997) 613.

[19] G. Gotsman, E. Levin, U. Maor, E. Naftali and A. Prygarin, Proc. of the Workshop on HERA and the LHC, part A, p. 221 (2005).

[20] A. Aktas et al. [H1 Collaboration], Eur. Phys. J. C 51 (2007) 549 [arXiv:hep-ex/0703022]. 